\title{
On the Importance of Displacement History in Soft-Body Contact Models
}

\author{
Jonathan Fleischmann; Radu Serban, Dan Negrut \\ Simulation Based Engineering Laboratory (SBEL) \\ Department of Mechanical Engineering, University of Wisconsin-Madison \\ Madison, Wisconsin 53706 \\ Emails: $\{$ jafleisc, serban, negrut $\} @$ wisc.edu \\ Paramsothy Jayakumar \\ US Army Tank Automotive Research Development and Engineering Center (TARDEC) \\ Warren, Michigan, 48397 \\ Email: paramsothy.jayakumar.civ@mail.mil
}

Two approaches are commonly used for handling frictional contact within the framework of the Discrete Element Method (DEM). One relies on the Complementarity Method (CM) to enforce a non-penetration condition and the Coulomb dry-friction model at the interface between two bodies in mutual contact. The second approach, called the Penalty Method (PM), invokes an elasticity argument to produce a frictional contact force that factors in the local deformation and relative motion of the bodies in contact. We give a brief presentation of a DEM-PM contact model that includes multi-time-step tangential contact displacement history. We show that its implementation in an open source simulation capability called Chrono is capable of accurately reproducing results from physical tests typical of the field of geomechanics; i.e., a direct shear tests on a mono-disperse material. Keeping track of the tangential contact displacement history emerges as a key element of the model. We show that identical simulations using contact models that include either no tangential contact displacement history or only single-time-step tangential contact displacement history are unable to accurately model the direct shear test.

\section{The Discrete Element Method}

Two alternative approaches have emerged as viable solutions for large frictional contact problems in granular flow dynamics and quasi-static geomechanics applications. The so-called Complementarity Method (CM) is generally favored within the multibody dynamics community, see for instance [1]. In this approach, individual particles in a bulk granular material are modeled as rigid bodies, and nonpenetration conditions are written as complementarity equations which, in conjunction with a Coulomb friction law, lead to a Differential Variational Inequality (DVI) form of the

\footnotetext{
${ }^{*}$ Corresponding author
}

Newton-Euler equations of motion [2]. Not limited by stability considerations, DEM-CM allows for much larger time integration steps than the alternative Penalty Method-based (PM) solutions, since the latter involve large contact stiffnesses that impose strict stability conditions on all explicit time integration algorithms. However, DEM-CM involves a relatively complex and computationally costly solution sequence per time step, since it leads to a mathematical program with complementarity and equality constraints, which must be relaxed to obtain tractable linear complementarity or cone complementarity problems [3].

More mature and widely adopted within the geomechanics community [4], DEM-PM can be viewed either as a regularization (or smoothing) approach, which relies on a relaxation of the rigid-body assumption, or as a deformable-body approach localized to the points of contact between individual particles in a bulk granular material $[5,6]$. In this approach, normal and tangential contact forces are calculated using various laws [7,8], which are based on the local body deformation at the point of contact. In the contact-normal direction, this local body deformation is defined as the penetration (overlap) of the two quasi-rigid bodies. In the tangential direction, the deformation is defined as the total tangential displacement incurred since the initiation of contact. Once contact forces are known, the time evolution of each body in the system is obtained by integrating the NewtonEuler equations of motion. Since in this approach the contact force-displacement law is derived from the elastic properties of the materials constituting the contacting bodies; i.e., Young's modulus and Poisson's ratio, the DEM-PM is capable of resolving statically indeterminate loading conditions that can exist at the particle level in random granular packings [9-11]. However, due to large contact stiffnesses and the use of explicit time integration [12], the DEM-PM approach is limited to very small time integration step-sizes to 


\section{Report Documentation Page}

Form Approved

OMB No. 0704-0188

Public reporting burden for the collection of information is estimated to average 1 hour per response, including the time for reviewing instructions, searching existing data sources, gathering and maintaining the data needed, and completing and reviewing the collection of information. Send comments regarding this burden estimate or any other aspect of this collection of information,

including suggestions for reducing this burden, to Washington Headquarters Services, Directorate for Information Operations and Reports, 1215 Jefferson Davis Highway, Suite 1204, Arlington

VA 22202-4302. Respondents should be aware that notwithstanding any other provision of law, no person shall be subject to a penalty for failing to comply with a collection of information if it

does not display a currently valid OMB control number.

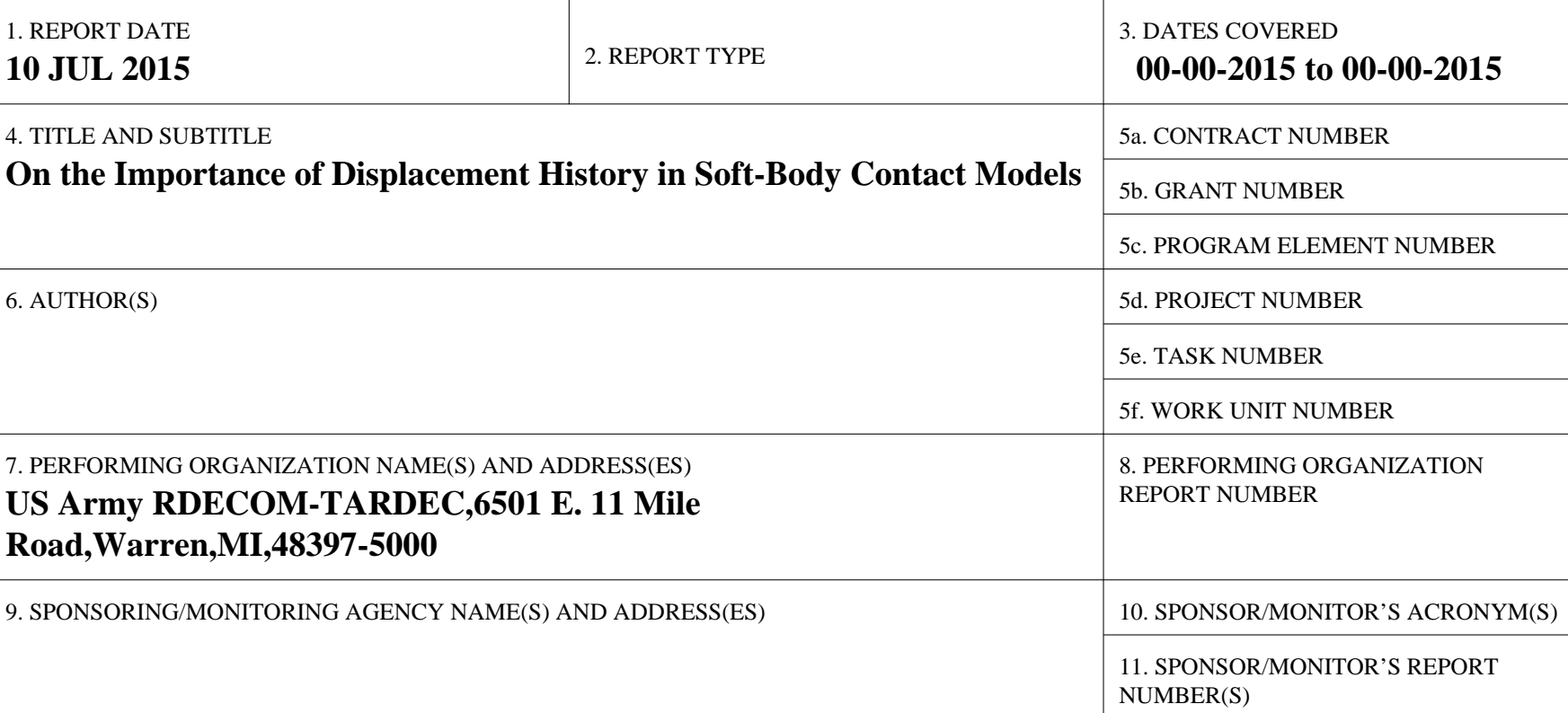

12. DISTRIBUTION/AVAILABILITY STATEMENT

Approved for public release; distribution unlimited

13. SUPPLEMENTARY NOTES

14. ABSTRACT

See Report

15. SUBJECT TERMS

16. SECURITY CLASSIFICATION OF:

\begin{tabular}{c|c|c|c}
$\begin{array}{c}\text { a. REPORT } \\
\text { unclassified }\end{array}$ & $\begin{array}{c}\text { b. ABSTRACT } \\
\text { unclassified }\end{array}$ & $\begin{array}{c}\text { c. THIS PAGE } \\
\text { unclassified }\end{array}$ & $\begin{array}{c}\text { Same as } \\
\text { Report (SAR) }\end{array}$ \\
\hline
\end{tabular}

\begin{tabular}{l|l}
$\begin{array}{c}\text { 18. NUMBER } \\
\text { OF PAGES } \\
\mathbf{6}\end{array}$ & 19a. NAME OF \\
& RESPONSIBLE PERSON \\
&
\end{tabular}

Standard Form 298 (Rev. 8-98) 
ensure stability.

\section{The Penalty Method or Soft-Body Approach}

A granular or particulate medium problem is modeled in DEM using a massive collection of distinct rigid or deformable elements having simple shapes that in many cases are spheres. In the DEM-PM or soft-body approach, the elements are "soft" - they are allowed to "overlap" or experience local deformation before a corrective contact force is applied at the point of contact. Once such an overlap $\delta_{n}$ is detected, by any one of a number of contact algorithms, contact force vectors $\mathbf{F}_{n}$ and $\mathbf{F}_{t}$ normal and tangential to the contact plane at the point of contact are calculated using various constitutive laws [7,8] based on the local body deformation at the point of contact. In the contact-normal direction, $\mathbf{n}$, this local body deformation is defined as the penetration (overlap) of the two quasi-rigid bodies, $\mathbf{u}_{n}=\delta_{n} \mathbf{n}$. In the contact-tangential direction, the deformation is defined as the total tangential displacement incurred since the initiation of contact, which is approximated as a vector $\mathbf{u}_{t}$ in the contact plane.

An example of a DEM-PM contact constitutive law, a slightly modified form of which is used in the open-source codes Chrono [13] and LIGGGHTS [14], is the following viscoelastic model based on either Hookean or Hertzian contact theory:

$$
\begin{aligned}
& \mathbf{F}_{n}=f\left(\bar{R}, \boldsymbol{\delta}_{n}\right)\left(k_{n} \mathbf{u}_{n}-\gamma_{n} \bar{m} \mathbf{v}_{n}\right) \\
& \mathbf{F}_{t}=f\left(\bar{R}, \delta_{n}\right)\left(-k_{t} \mathbf{u}_{t}-\gamma_{t} \bar{m} \mathbf{v}_{t}\right),
\end{aligned}
$$

where $\mathbf{u}=\mathbf{u}_{n}+\mathbf{u}_{t}$ is the overlap or local contact displacement of two interacting bodies, see Fig. 1. The quantities $\bar{m}=m_{i} m_{j} /\left(m_{i}+m_{j}\right)$ and $\bar{R}=R_{i} R_{j} /\left(R_{i}+R_{j}\right)$ represent the effective mass and effective radius of curvature, respectively, for contacting bodies with masses $m_{i}$ and $m_{j}$ and contact radii of curvature $R_{i}$ and $R_{j}$. The relative velocity at the contact point, $\mathbf{v}=\mathbf{v}_{n}+\mathbf{v}_{t}$, and its normal and tangential components $\mathbf{v}_{n}$ and $\mathbf{v}_{t}$ are computed as

$$
\begin{aligned}
\mathbf{v} & =\mathbf{v}_{j}+\Omega_{j} \times \mathbf{r}_{j}-\mathbf{v}_{i}-\Omega_{i} \times \mathbf{r}_{i} \\
\mathbf{v}_{n} & =(\mathbf{n} \cdot \mathbf{v}) \mathbf{n} \\
\mathbf{v}_{t} & =\mathbf{v}-\mathbf{v}_{n},
\end{aligned}
$$

where $\mathbf{v}_{i}$ and $\mathbf{v}_{j}$ are the velocity vectors of the centers of mass of bodies $i$ and $j, \Omega_{i}$ and $\Omega_{j}$ are the angular velocity vectors of bodies $i$ and $j$, and $\mathbf{r}_{i}$ and $\mathbf{r}_{j}$ are the position vectors from the centers of mass of bodies $i$ and $j$ to the point of contact. For Hookean contact, $f\left(\bar{R}, \delta_{n}\right)=1$ in Eqn. (1); for Hertzian contact, one can let $f\left(\bar{R}, \delta_{n}\right)=\sqrt{\bar{R} \delta_{n}}[15,16]$. The normal and tangential stiffness and damping coefficients $k_{n}$, $k_{t}, \gamma_{n}$, and $\gamma_{t}$ are obtained, through various constitutive laws derived from contact mechanics, from physically measurable properties for the materials of the contacting bodies, such as Young's modulus, Poisson's ratio, the coefficient of restitu- tion, etc. Detailed descriptions of the contact models implemented in Chrono and LIGGGHTS, as well as alternative contact models are provided in [17].

The component of the overlap or contact displacement vector $\mathbf{u}$ in the contact-normal direction, $\mathbf{u}_{n}=\delta_{n} \mathbf{n}$, is obtained directly from the contact detection algorithm, which provides the magnitude of the "inter-penetration" $\delta_{n}$. It follows that $\mathbf{u}_{n}$ is parallel to the normal component of the relative velocity vector $\mathbf{v}_{n}$ at the point of contact. However, it is important to note that, in general, the same is not true of the tangential component of the overlap vector, or tangential contact displacement, $\mathbf{u}_{t}$ and the tangential component of the relative velocity vector $\mathbf{v}_{t}$. Specifically, they must lie in the contact plane, but may or may not be parallel to each other. In particular, even if there is no relative tangential velocity at the contact point, there may still be a tangential contact force induced by deformation in the tangential plane, and this force may be needed to support static friction.

Herein, the tangential contact displacement vector $\mathbf{u}_{t}$ is formulated as

$$
\mathbf{u}_{t}=\int_{t_{0}}^{t} \mathbf{v}_{t} d t-\left(\mathbf{n} \cdot \int_{t_{0}}^{t} \mathbf{v}_{t} d t\right) \mathbf{n}
$$

where $t$ is the current time and $t_{0}$ is the time at the initiation of contact. For the true tangential contact displacement history model, the vector $\mathbf{u}_{t}$ must be stored and updated at each time step for each contact point on a given pair of contacting bodies from the time that contact is initiated until that contact is broken. The tangential (or shear) contact displacement history vector is then given at time step $i$ by

$$
\begin{aligned}
\mathbf{u}_{t, i}^{*} & =\mathbf{u}_{t, i-1}+\mathbf{v}_{t, i} \Delta t_{i} \\
\mathbf{u}_{t, i} & =\mathbf{u}_{t, i}^{*}-\left(\mathbf{n}_{i} \cdot \mathbf{u}_{t, i}^{*}\right) \mathbf{n}_{i},
\end{aligned}
$$

where $\Delta t_{i}$ is the integration time step size, $t_{i}=t_{i-1}+\Delta t_{i}$, and a subscript indicates the time step at which each variable is evaluated. The projection of $\mathbf{u}_{t}^{*}$ onto the contact plane is necessary to ensure that $\mathbf{u}_{t}$ is in the contact plane at each time step. Note that $\mathbf{u}_{t, k-1}$ is set to zero at the initiation of contact, for some $k$.

A simpler but less effective tangential contact displacement model suggested in the literature is a single time step approximation of Eqn. (4), given for any time step by

$$
\mathbf{u}_{t}=\mathbf{v}_{t} \Delta t .
$$

This model, which we will call pseudo-history, essentially assumes that contact never persists for more than a single time step, and it is unable to support a static friction force in the absence of relative tangential velocity.

To enforce the Coulomb friction law, if $\left|\mathbf{F}_{t}\right|>\mu\left|\mathbf{F}_{n}\right|$ at any given time step, then before the contributions of the contact forces are added to the resultant force and torque on the body, the (stored) value of $\left|\mathbf{u}_{t}\right|$ is scaled so that $\left|\mathbf{F}_{t}\right|=\mu\left|\mathbf{F}_{n}\right|$, 

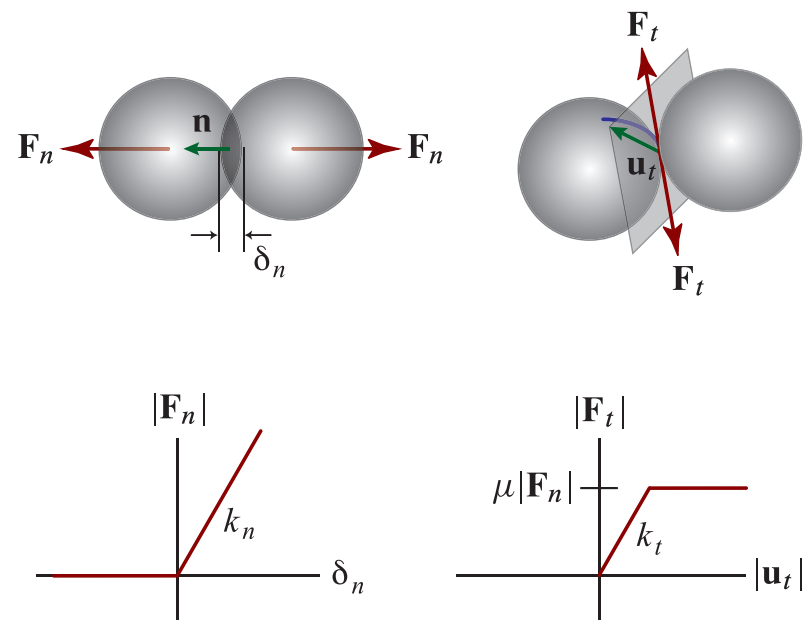

Fig. 1. DEM-PM contact model described in this section, with normal overlap distance $\delta_{n}$, contact-normal unit vector $\mathbf{n}$, and tangential displacement vector $\mathbf{u}_{t}$ in the plane of contact (top), and with a Hookean-linear contact force-displacement law with constant Coulomb sliding friction (bottom).

where $\mu$ is the Coulomb (static and sliding) friction coefficient. For example, if $f(x)=1$ in Eqn. (1), then

$$
k_{t}\left|\mathbf{u}_{t}\right|>\mu\left|\mathbf{F}_{n}\right| \quad \Rightarrow \quad \mathbf{u}_{t} \leftarrow \mathbf{u}_{t} \frac{\mu\left|\mathbf{F}_{n}\right|}{k_{t}\left|\mathbf{u}_{t}\right|}
$$

Figure 1 illustrates the DEM-PM contact model described in this section with a Hookean-linear contact forcedisplacement law with constant Coulomb sliding friction. Once the contact forces $\mathbf{F}_{n}$ and $\mathbf{F}_{t}$ are computed for each contact and their contributions are summed to obtain a resultant force and torque on each body in the system, the time evolution of each body in the system is obtained by integrating the Newton-Euler equations of motion, subject to the Courant-Friedrichs-Lewy (CFL) stability condition, which requires [18] that $\Delta t<\Delta t_{\text {crit }} \sim \sqrt{m_{\min } / k_{\max }}$.

\section{The Importance of Multi-Step Tangential Contact Displacement History}

To demonstrate the importance of using tangential displacement history in the DEM-PM contact model, we first perform direct shear simulations of small randomly packed specimens of 1,800 and 5,000 identical spheres in Chrono [13] and LIGGGHTS [14]. The inside dimensions of the shear box are $6 \mathrm{~cm}$ in length by $6 \mathrm{~cm}$ in width, and the height of the granular material specimen is also approximately $6 \mathrm{~cm}$. The spheres have a uniform diameter of $5 \mathrm{~mm}$. The random packing of 1,800 spheres was initially obtained by a "rainfall" method, after which the spheres were compacted with friction temporarily turned off to obtain a dense packing. The resulting void ratio was approximately $e=0.4$, which corresponds to a dense packing [19,20]. For this comparison, the material properties for spheres were taken to be those corresponding to quartz - the density is $2,500 \mathrm{~kg} / \mathrm{m}^{3}$,

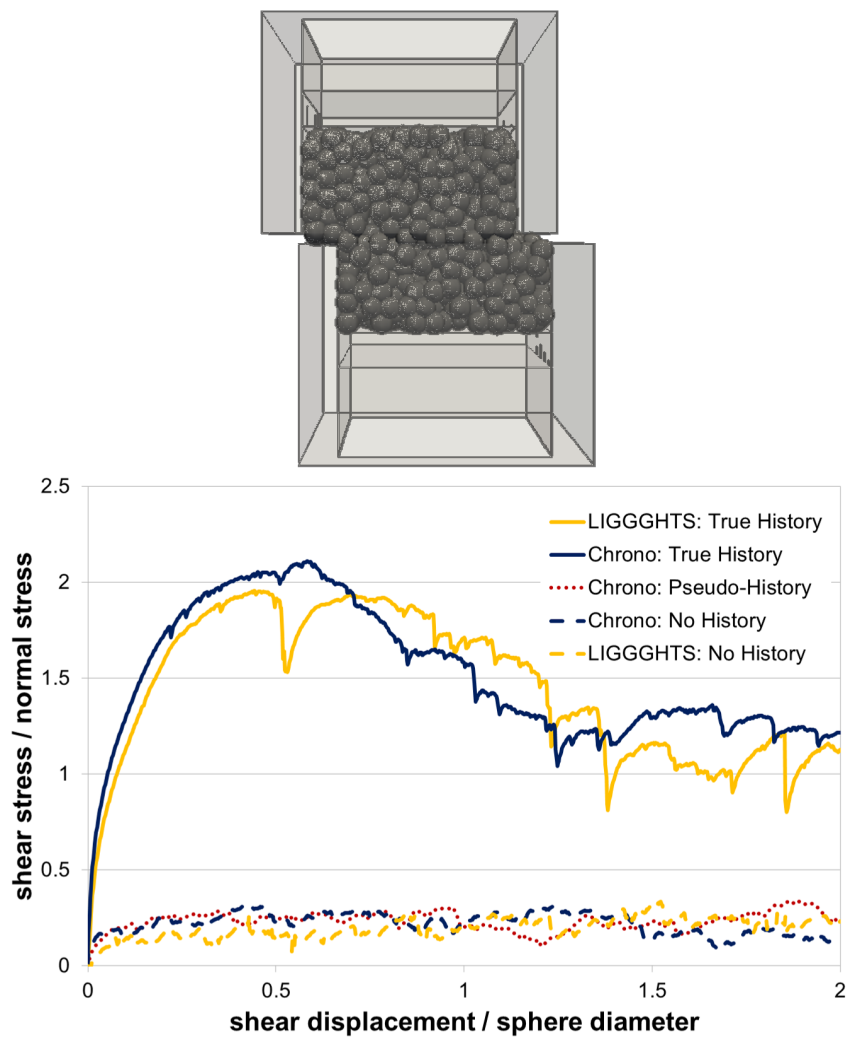

Fig. 2. Direct shear simulation setup (top) and shear versus displacement results (bottom) obtained by Chrono [13] and LIGGGHTS [14] for 1,800 randomly packed uniform spheres using the true tangential contact displacement history model of Eqn. (4), the pseudo-history model of Eqn. (5), and no tangential contact history.

the inter-particle friction coefficient is $\mu=0.5$, Poisson's ratio is $v=0.3$, and the elastic modulus is $E=8\left(10^{10}\right) \mathrm{Pa}$. However, in order to ensure a stable simulation with a reasonable time integration step-size of $\Delta t=10^{-5} \mathrm{~s}$, the elastic modulus was reduced by four orders of magnitude to $E=8\left(10^{6}\right) \mathrm{Pa}$. The shear speed was $1 \mathrm{~mm} / \mathrm{s}$. The simulation geometry in its final position is shown in Fig. 2 (top). Figure 2 (bottom) shows the shear-displacement curves obtained by Chrono and LIGGGHTS with the same normal and tangential contact force-displacement models. The labels "True History" and "No History" refer to whether or not tangential contact history is stored and used in the friction model. Included in this comparison is the "Pseudo-History" scenario, in which the tangential contact displacement vector is approximated by the product of the relative tangential velocity vector at the contact point and the time step-size at any given time. This pseudo-history approach is attractive, since unlike the "True History" alternative, it avoids the storage of a tangential contact history vector over multiple time steps for each contact point. However, Fig. 2 shows that the pseudo-history approximation is no better than ignoring tangential displacement history altogether for the quasi-static direct shear test. This is explained by the observation that under quasi-static (or static) deformation conditions, the de- 
pendence of the pseudo-history approximation on the relative inter-particle tangential velocity, which is zero, effectively eliminates the inter-particle tangential contact force and so renders the inter-particle friction coefficient $\mu$ effectively zero.

Also noteworthy in Fig. 2 is the fact that the interparticle friction coefficient $\mu$ for the spheres, which could also be described as a micro-scale "inter-particle friction angle" $\phi_{\mu}=\tan ^{-1} \mu$, is nowhere close to having the same value as the macro-scale "material friction coefficient" $\mu_{\text {macro }}$ for the bulk granular material. The latter, more commonly described as a bulk granular material friction angle $\phi=$ $\tan ^{-1} \mu_{\text {macro }}$, is the material parameter that defines the yield surface for the bulk granular material according to the MohrCoulomb yield criterion. The material friction angle $\phi$ is also known as the angle of repose for the bulk granular material. Nor should it be surprising that $\phi \neq \phi_{\mu}$, since, as noted in [21], even if the inter-particle friction coefficient $\mu$, and hence the micro-scale friction angle $\phi_{\mu}$, is zero, the bulk granular material friction angle $\phi$ will in general not be zero. Rather, if $\mu=0$, then $\phi=\psi$, where $\psi$ is the dilation angle of the granular material. Note that typically, $\psi \approx 15^{\circ}$ for densely packed well-graded sands [22]. In particular, we note from Fig. 2 that, when the tangential contact displacement history model is used, while $\mu=0.5$ and hence $\phi_{\mu} \approx 26.6^{\circ}$ for the spheres, the peak ratio of shear stress to normal stress for the bulk granular material is $\mu_{\text {macro }} \approx 2$, and hence $\phi_{p} \approx 63^{\circ}$; and the residual ratio of shear stress to normal stress for the bulk granular material is $\mu_{\text {macro }} \approx 1$, and hence $\phi_{r} \approx 45^{\circ}$. On the other hand, when the tangential contact displacement history model is not used, $\mu_{\text {macro }} \approx 0.25$ throughout the simulation, and hence $\phi_{p}=\phi_{r}=\phi \approx 14^{\circ}$. Note that all of these results are obtained in the absence of any rolling or spinning friction.

To emphasize the importance of using multi-step tangential contact displacement history, it should be pointed out that other factors involved in the model, such as the values of $k_{n}, k_{t}, \gamma_{n}$, and $\gamma_{t}$, turned out to play a secondary role in the outcome of the simulation. In fact, a significant degree of variation exists in the literature for the exact values of the contact stiffness coefficients $k_{n}$ and $k_{t}$ [17]. The same is true for the mass proportional damping coefficients $\gamma_{n}$ and $\gamma_{t}$. The latter are frequently simply chosen sufficiently large to eliminate numerical noise in the DEM-PM simulations. For example, the results of DEM-PM simulations of direct (ring) shear tests with periodic boundary conditions on ASTM C 778-06 standard graded (quartz) sand with a log-normal particle size distribution, mean diameter $D_{50}=0.35 \mathrm{~mm}$, and coefficient of uniformity $C_{u}=1.7$ were considered in [23]. In these simulations, which employed the multi-step tangential contact displacement history model described herein, the damping coefficients in Eqn. (1) were taken to be $\gamma_{n}=40 \mathrm{~s}^{-1}$ and $\gamma_{t}=20 \mathrm{~s}^{-1}$, and the contact stiffnesses $k_{n}$ and $k_{t}$ were taken to be constant, with $k_{n}=10^{12} \mathrm{~N} / \mathrm{m}$ and $k_{t}=8\left(10^{11}\right)$ $\mathrm{N} / \mathrm{m}$. Despite these simplifications, and the fact that the simulations performed included no rolling friction and the sand particles were modeled as spheres of different sizes, the correct macro-scale residual bulk granular material friction an- gle of $\phi_{r}=30^{\circ}$ [24] was reproduced exactly. The only other material parameter that needed to be specified, in addition to the particle size distribution, was the inter-particle friction coefficient $\mu=0.5$, which is considered by Mitchell and Soga [20] to be "reasonable for quartz, both wet and dry." Note that the values of the peak and residual friction angles are strongly dependent on the particle size distribution [25], which is why the residual friction angle for uniform quartz spheres cannot be expected to be the same as that of quartz spheres (or well-rounded quartz sand) with a log-normal particle size distribution.

\section{Validation Against Direct Shear Experiments With Uniform Glass Beads}

Whereas the previous section demonstrated the difference in results between the "True History" and "No History" scenarios, herein we compare the "True History" shear-displacement curves against experimental data reported in [26]. Specifically, to verify that the Chrono DEM-PM contact model with true tangential displacement history currently does indeed accurately model the microscale physics and emergent macro-scale properties of a simple granular material, Fig. 3 shows shear versus displacement curves obtained from both experimental [26] (top) and Chrono-simulated (center and bottom) direct shear tests, performed under constant normal stresses of 3.1, 6.4, 12.5, and $24.2 \mathrm{kPa}$, on 5,000 uniform glass beads. The simulation geometry in its final position is similar to that shown in Fig. 2 (top), except that the inside dimensions of the shear box are now $12 \mathrm{~cm}$ in length by $12 \mathrm{~cm}$ in width. The height of the granular material specimen in the box is still approximately $6 \mathrm{~cm}$. In both the experimental and simulated direct shear tests, the glass spheres have a uniform diameter of $6 \mathrm{~mm}$, and the random packing of 5,000 spheres was initially obtained by a "rainfall" method, after which the spheres were compacted by the confining normal stress without adjusting the inter-particle friction coefficient. The DEM-PM simulations were performed in Chrono using a Hertzian normal contact force model and true tangential contact displacement history with Coulomb friction. The material properties of the spheres in the simulations were taken to be those corresponding to glass [26], for which the density is $2,550 \mathrm{~kg} / \mathrm{m}^{3}$, the inter-particle friction coefficient is $\mu=0.18$, Poisson's ratio is $v=0.22$, and the elastic modulus is $E=4\left(10^{10}\right) \mathrm{Pa}$, except that the elastic modulus was again reduced by several orders of magnitude, to $E=4\left(10^{6}\right) \mathrm{Pa}$ (center) and $E=4\left(10^{7}\right) \mathrm{Pa}$ (bottom) for comparison, to ensure a stable simulation with a reasonable time integration step-size of $\Delta t=10^{-5} \mathrm{~s}$. The shear speed was $1 \mathrm{~mm} / \mathrm{s}$.

Figure 3 (center) shows that the DEM-PM direct shear simulations performed in Chrono on 5,000 glass spheres with $E=4\left(10^{6}\right) \mathrm{Pa}$ matches reasonably well the physical experiments for all but the highest normal stress of $24.2 \mathrm{kPa}$. This observed error in the simulation results, which increases with increasing normal stress, is consistent with the fact that the contact stiffness for the spheres in these DEM-PM simulations is four orders of magnitude smaller than the stiffness 

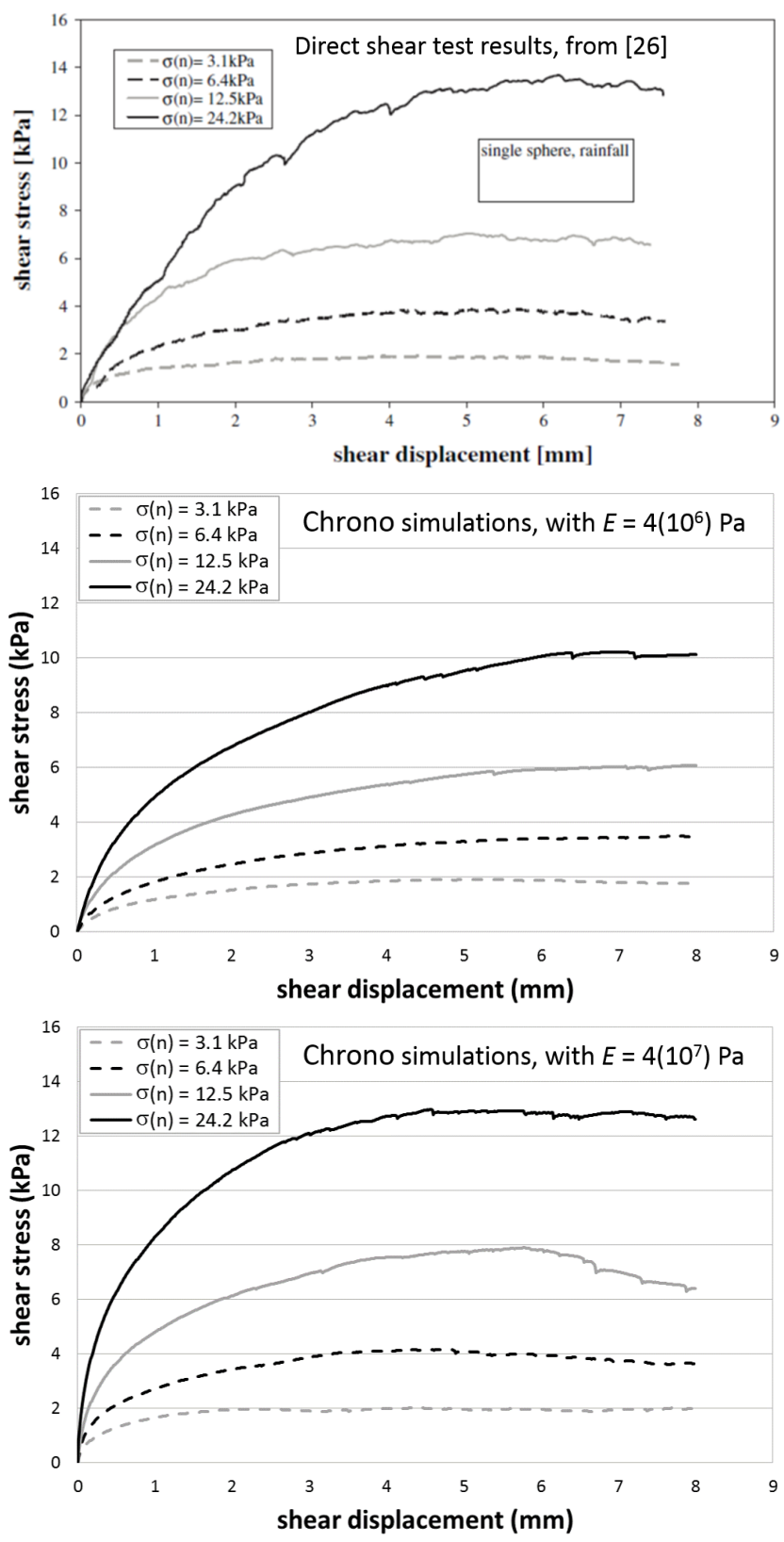

Fig. 3. Direct shear test results for 5,000 randomly packed uniform glass beads obtained by experiment [26] (top) and DEM-PM simulations using Chrono (center and bottom), under constant normal stresses of $3.1,6.4,12.5$, and $24.2 \mathrm{kPa}$. For the DEM-PM simulations, elastic moduli of $E=4\left(10^{6}\right) \mathrm{Pa}$ (center) and $E=4\left(10^{7}\right) \mathrm{Pa}$ (bottom) are used.

of true glass beads. To explore the effect that the value of the elastic modulus has on the DEM-PM direct shear results, we have also performed DEM-PM simulations using an elastic modulus of $E=4\left(10^{7}\right) \mathrm{Pa}$ for the spheres, which is still three orders of magnitude smaller than the true elastic modulus of glass beads. Figure 3 (bottom) shows that increasing the value of the elastic modulus of the spheres in the direct shear simulations by an order of magnitude to $E=4\left(10^{7}\right) \mathrm{Pa}$; i.e., using a contact stiffness for the spheres that is three rather than four orders of magnitude smaller than the physically correct contact stiffness, results in a peak and residual shear stress that is much closer to the experimentally observed values for all four of the constant normal stresses tested. This is a significant observation, since it has often been argued in the DEM-PM literature that decreasing the value of the elastic modulus to allow a larger stable time step-size should only affect the elastic portion of the shear displacement curve for the bulk granular material. A comparison of Figs. 3 (center) and 3 (bottom), however, while confirming this difference in the elastic portion of the shear-displacement curve, also reveals a significant difference in the plastic or post-yield portion of the shear-displacement curve for the direct shear test, in particular the peak and residual shear stresses, and the corresponding peak and residual friction angles, for all four of the constant normal stresses tested.

\section{Conclusions}

In relation to using computer simulation to capture the dynamics of granular material, this technical note makes the following two points. First and foremost, contrary to common perception, eliminating the tangential contact history in DEM-PM yields wrong results in a shear test that, while basic and deceptively simple, remains difficult to simulate. Moreover, a quasi-history approach that only relies on the tangential deformation at the current time step produces inaccurate results. Second, a comparison against experimental data suggests that the simulation results are only moderately impacted by the values selected for the DEM-PM model parameters, of which the normal stiffness $k_{n}$ turns out to quantitatively influence the most the outcome of the numerical experiments. Specifically, over a broad spectrum of values for $k_{t}, \gamma_{n}$, and $\gamma_{t}$, the simulation results are qualitatively acceptable for artificially low values of $k_{n}$, a compromise made in order to allow stable numerical integration at larger simulation time steps. However, more accurate results call for higher values of $k_{n}$ that come close to the theory predicted values for this parameter.

\section{Acknowledgements}

This research was supported in part by US Army Rapid Innovation Fund No. W911NF-13-R-0011, Topic No. 6a Maneuverability Prediction.

\section{References}

[1] Tasora, A., and Anitescu, M., 2010. "A convex complementarity approach for simulating large granular flows". Journal of Computational and Nonlinear Dynamics, 5(3), pp. 1-10.

[2] Stewart, D. E., 2000. "Rigid-body dynamics with friction and impact". SIAM Review, 42(1), pp. 3-39.

[3] Anitescu, M., and Tasora, A., 2010. "An iterative approach for cone complementarity problems for nonsmooth dynamics". Computational Optimization and Applications, 47(2), pp. 207-235. 
[4] O'Sullivan, C., 2011. "Particle-based discrete element modeling: Geomechanics perspective". Int. J. Geomech., 11(6), pp. 449-464.

[5] Cundall, P., and Strack, O., 1979. "A discrete element model for granular assemblies". Geotechnique, 29, pp. 47-65.

[6] Pöschel, T., and Schwager, T., 2005. Computational granular dynamics: models and algorithms. Springer.

[7] Kruggel-Emden, H., Simsek, E., Rickelt, S., Wirtz, S., and Scherer, V., 2007. "Review and extension of normal force models for the discrete element method". Powder Technology, 171, pp. 157-173.

[8] Kruggel-Emden, H., Wirtz, S., and Scherer, V., 2008. "A study of tangential force laws applicable to the discrete element method (DEM) for materials with viscoelastic or plastic behavior". Chem. Eng. Sci., 63, pp. 1523-1541.

[9] Fleischmann, J. A., Drugan, W. J., and Plesha, M. E., 2013. "Direct micromechanics derivation and DEM confirmation of the elastic moduli of isotropic particulate materials, Part I: No particle rotation". J. Mech. Phys. Solids, 61(7), pp. 1569-1584.

[10] Fleischmann, J. A., Drugan, W. J., and Plesha, M. E., 2013. "Direct micromechanics derivation and DEM confirmation of the elastic moduli of isotropic particulate materials, Part II: Particle rotation". J. Mech. Phys. Solids, 61(7), pp. 1585-1599.

[11] Kruyt, N. P., 2014. "Micromechanical study of elastic moduli of three-dimensional granular assemblies". Int. J. Solids Struct., 51, pp. 2336-2344.

[12] Samiei, K., Peters, B., Bolten, M., and Frommer, A., 2013. "Assessment of the potentials of implicit integration method in discrete element modelling of granular matter". Computers \& Chemical Engineering, 49, pp. 183-193.

[13] Chrono Website. http: / / projectchrono.org/ chronoengine/. Accessed: 2015-03-19.

[14] LIGGGHTS Website. http: / / www. cfdem. com/. Accessed: 2015-03-19.

[15] Silbert, L. E., Ertaş, D., Grest, G. S., Halsey, T. C., Levine, D., and Plimpton, S. J., 2001. "Granular flow down an inclined plane: Bagnold scaling and rheology". Physical Review E, 64(5), p. 051302.

[16] Zhang, H. P., and Makse, H. A., 2005. "Jamming transition in emulsions and granular materials". Physical Review E, 72(1), p. 011301.

[17] Fleischmann, J., 2015. DEM-PM Contact Model with Multi-Step Tangential Contact Displacement History. Tech. Rep. TR-2015-06: http://sbel . wisc.edu/documents/TR-2015-06.pdf,

Simulation-Based Engineering Laboratory, University of Wisconsin-Madison.

[18] O’Sullivan, C., and Bray, J. D., 2004. "Selecting a suitable time step for discrete element simulations that use the central difference time integration scheme". Engineering Computations, 21(2-4), pp. 278-303.

[19] Bardet, J.-P., 1997. Experimental Soil Mechanics. Prentice Hall.
[20] Mitchell, J. K., and Soga, K., 2005. Fundamentals of Soil Behavior. John Wiley and Sons.

[21] Fleischmann, J. A., 2013. "Micromechanics-based continuum constitutive modeling of isotropic noncohesive particulate materials, informed and validated by the discrete element method". PhD thesis, Department of Engineering Mechanics, University of Wisconsin-Madison.

[22] Vermeer, P. A., and de Borst, R., 1984. "Nonassociated plasticity for soils, concrete and rock". Heron, 29(3), pp. 1-64.

[23] Fleischmann, J. A., Plesha, M. E., and Drugan, W. J., 2013. "Quantitative comparison of two-dimensional and three-dimensional discrete element simulations of nominally two-dimensional shear flow". Int. J. Geomech., 13(3), pp. 205-212.

[24] Cho, G.-C., Dodds, J., and Santamarina, J. C., 2006. "Particle shape effects on packing density, stiffness, and strength: Natural and crushed sands". J. Geotech. Geoenviron. Eng., 132(5), pp. 591-602.

[25] Cheng, Y. P., and Minh, N. H., 2009. "DEM investigation of particle size distribution effect on direct shear behaviour of granular agglomerates". In Powders and Grains 2009, Proceedings of the 6th International Conference on Micromechanics of Granular Media, pp. 401-404.

[26] Härtl, J., and Ooi, J. Y., 2008. "Experiments and simulations of direct shear tests: porosity, contact friction and bulk friction". Granular Matter, 10(4), pp. 263271. 